\title{
EVALUACIÓN DE LAS TÉCNICAS DE EXTRACCIÓN DE PIEZAS FRACTURADAS EN EL TRANSQUIRÚRGICO
}

\author{
Génesis Lisbeth Ferrand-Peña ${ }^{*}$, Est. ${ }_{1}$, Giancarlo García-Castillo, Est. ${ }_{1}$, \\ Johanny Stephany Castillo-Medrano, Est. ${ }_{1}$, Amelia Mercedes Cáceres-Tejada, Est. ${ }_{1}$, \\ Patricia Grau-Gullón, M.Sc. ${ }_{1}$, Laura Reyes-Alardo, Odont. ${ }_{1}$
}

${ }_{1}$ Facultad de Odontología, Universidad Iberoamericana (Unibe), República Dominicana

Recibido: 20 de agosto 20 del 2013. Aprobado: 25 de septiembre del 2013.

*Autor de correspondencia: Génesis Lisbeth Ferrand Peña, Escuela de Odontología, Facultad de Ciencias de la Salud, Universidad Iberoamericana (Unibe), (809) 689 4111, Ave. Francia № 129, Gazcue, Santo Domingo, República Dominicana, correos electrónicos: info@unibe.edu.do, patricia.grau@gmail.com.

Cómo citar este artículo: Ferrand-Peña GL, García-Castillo G, Castillo-Medrano JS, Cáceres-Tejada AM, Grau-Gullón P, Reyes-Alardo L. Evaluación de las técnicas de extracción de piezas fracturadas en el transquirúrgico. Rev Nac Odontol. 2013; 9(17): 47-51.

Resumen. Introducción: el objetivo de este trabajo de investigación fue explicar cuáles son las técnicas más utilizadas para extraer una pieza que se fractura en el transquirúrgico, en el área de cirugía de la Universidad Iberoamericana, durante el período febrero-mayo del 2013, y demostrar si los estudiantes del pregrado son capaces o no de resolver este tipo de complicación. Métodos: para este estudio se diseñó un cuestionario, el cual fue dispuesto en el área de cirugía de la clínica dental de la Universidad Iberoamericana; los doctores responsables de cada tanda de cirugía debieron contestarlo y firmarlo al momento en que se fracturara una pieza durante el transquirúrgico. También fue realizada una encuesta a los doctores del área, para corroborar y obtener sus puntos de vista sobre los datos recolectados con el cuestionario. Resultados: la odontosección, el levantamiento de un colgajo, ostectomía y el uso de elevadores apicales son las técnicas utilizadas en esta área para extraer un fragmento fracturado. Conclusiones: se demostró que la odontosección fue la técnica más utilizada. A su vez se determinó que en la mayoría de los casos de fractura, el doctor responsable tuvo que intervenir en la extracción.

Palabras clave: elevadores dentales, fractura dental, levantamiento de un colgajo, odontosección, osteotomía, técnicas de extracción.

\section{Evaluation of Techniques Used to Extract Fractured Dental Pieces During Surgical Procedures}

Abstract. Introduction: The objective of this research was to explain techniques used to extract fractured dental pieces during surgical procedures in the surgery area of the Universidad Iberoamericana during the period from February to May of 2013, to determine whether or not undergraduate students are able to resolve such a complication. Methods: A questionnaire was designed for the study, which was then placed in the dental surgery area of the University; the doctors responsible for each surgery round had to fill out and sign the questionnaire whenever a piece was fractured. A survey was also carried out among the doctors in the area to verify and obtain their assessments of the data that had been obtained using the questionnaire. Results: Odontosection, flap lifting, osteotomy and apical elevators are the techniques used in this area to remove broken pieces. Conclusion: Odontosection was found to be the most frequently used technique. It was also determined that in most fracture cases, the responsible doctor had to intervene in the extraction.

Keywords: dental elevators, tooth fracture, flap lifting, osteotomy, extraction techniques.

\section{Avaliação das técnicas de extração de peças fraturadas no transcirúrgico}

Resumo. Introdução: o objetivo deste trabalho de pesquisa foi explicar quais são as técnicas mais utilizadas para extrair uma peça que se fratura no transcirúrgico, na área de cirurgia da Universidade Iberoamericana, durante o período fevereiro-março de 2013, e demonstrar se os estudantes da graduação são ou não capazes de resolver esse tipo de complicação. Métodos: para este estudo, desenhou-se um questionário, o qual foi disposto na área de cirurgia da clínica dental da Universidade Ibero-americana; os doutores responsáveis de cada período de cirurgia o responderam e assinaram no momento em que uma peça tenha sido fraturada durante o transcirúrgico. Também foi realizada uma enquete aos doutores da área para corroborar e obter seus pontos de vista sobre os dados coletados com o questionário. Resultados: a odontosecção, o levantamento de um gajo, ostectomia e o uso de elevadores apicais são as técnicas utilizadas nesta área para extrair um fragmento fraturado. Conclusões: demonstrou-se que a odontosecção foi a técnica mais utilizada. Por sua vez, determinou-se que, na maioria dos casos de fratura, o médico responsável teve que intervir na extração.

Palavras-chave: elevadores dentais, fratura dental, levantamento de um gajo, odontosecção, ostectomia, técnicas de extração. 


\section{Introducción}

La odontología moderna se enfoca en la preservación de las piezas dentarias el mayor tiempo posible en boca; sin embargo, la extracción dental sigue siendo una de las prácticas más realizadas en una consulta dental, y a pesar de que se realiza con frecuencia, tiende a enfrentarse con muchas complicaciones mientras es realizada. López [1] indica que el profesional de la odontología que realice la práctica debe conocer las técnicas que va a utilizar para la solución de este tipo de complicación.

$\mathrm{El}$ conocer las conductas que se deben seguir frente a una pieza fracturada garantiza el aumento de la confianza en sí mismo del operador, disminuye la inseguridad del paciente, evita traumatismos innecesarios y aumenta las posibilidades de garantizar un mejor posquirúrgico.

Este estudio ayudará no sólo a la Universidad Iberoamericana, sino también a otras universidades de la República Dominicana, a implementar técnicas eficaces de extracción, no sólo para que no se fracturen las piezas en el transquirúrgico, sino también para, en caso de que ocurra, formar profesionales capaces de salir airosos de este tipo de complicación.

Este estudio tiene como objetivo general explicar cuál es la técnica más utilizada para extraer una pieza que se fractura en el transquirúrgico en el área de cirugía de la Universidad Iberoamericana, durante el periodo febrero-mayo del 2013.

\section{Métodos}

Para la realización de este estudio se contabilizaron todas las cirugías realizadas en el preclínico de la Universidad Iberoamericana, en el periodo febrero-mayo del 2013. Fueron 848 exodoncias simples y 168 exodoncias con levantamiento de colgajo (1.016 cirugías en total) registradas en esta área, realizadas por los estudiantes del pregrado de los niveles de clínica 2, 3, 4 y 5 . En estas cirugías se registraron 37 casos de fractura dental.

Para este estudio se diseñó un cuestionario (figura 1), el cual fue dispuesto en el área de cirugía de la clínica dental de la Universidad Iberoamericana, durante el período febrero-mayo del 2013. Los doctores responsables de cada tanda de cirugía debieron contestar y firmar el cuestionario al momento de que se fracturara una pieza en esta área. También fue realizada una encuesta (figura 2) a los doctores del área de cirugía de la Clínica de la Escuela de Odontología; su finalidad fue corroborar y conocer sus puntos de vista sobre los datos obtenidos con el cuestionario elaborado para recolectar los datos sobre las técnicas quirúrgicas para la extracción de piezas fracturadas en el transquirúrgico.

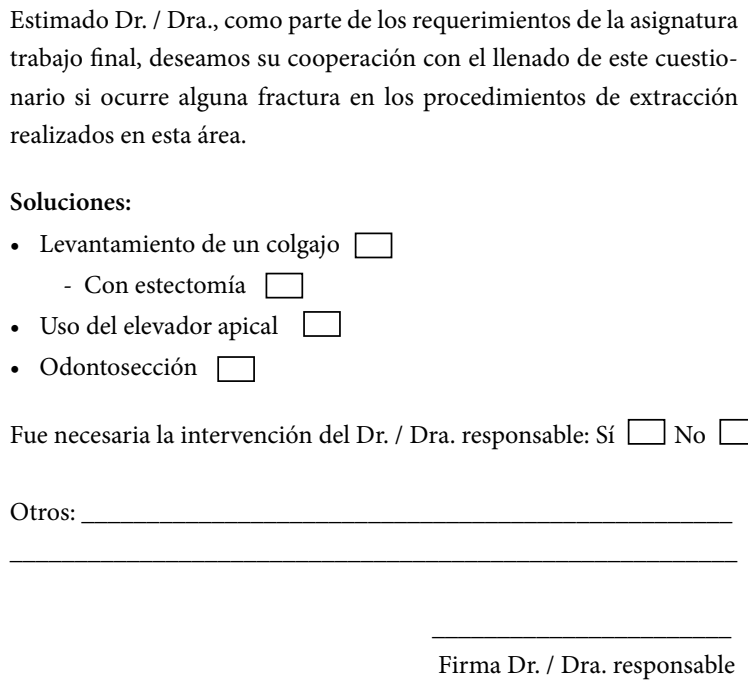

Estimado Dr. / Dra., como parte de los requerimientos de la asignatura trabajo final, deseamos su cooperación con el llenado de este cuestionario si ocurre alguna fractura en los procedimientos de extracción realizados en esta área.

Soluciones:

- Levantamiento de un colgajo

- Con estectomía

- Uso del elevador apical

- Odontosección

Fue necesaria la intervención del Dr. / Dra. responsable: Sí $\square$ No Otros:

Firma Dr. / Dra. responsable

Figura 1. Cuestionario

Fuente: elaboración propia

Estimado Dr. / Dra., como parte de los requerimientos de la asignatura trabajo final, deseamos su cooperación con el llenado de este cuestionario para conocer su punto de vista acerca de las causas que pueden provocar para la extracción de un fragmento fracturado.

1. ¿Qué factor o factores determinan la elección de una técnica o instrumento cuando se fractura una pieza dental en el trasquirúrgico?

2. ¿Son los estudiantes de la Universidad Iberoamericana capaces de resolver una complicaicón de este tipo?

Figura 2. Encuesta

Fuente: elaboración propia

\section{Resultados y discusión}

Los resultados de estos cuestionarios, correspondientes a la o las técnicas utilizadas para la extracción de los fragmentos fracturados, se describen a continuación.

El empleo de un levantamiento de colgajo con realización de una osteotomía fue necesario utilizarlo en el $21 \%$ de los casos en los que se fracturó una pieza dental en el transquirúrgico, en la clínica dental de la Universidad Iberoamericana, en el período febrero-mayo del 2013 (figura 3). 


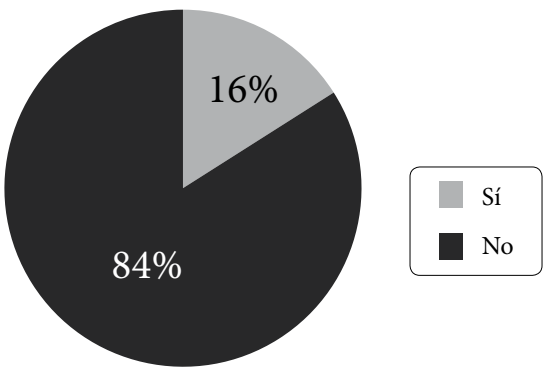

Figura 3. Levantamiento de un colgajo con ostectomía Fuente: elaboración propia

Estos resultados sustentan las opiniones sobre el empleo de esta técnica en los estudios de Irureta [2], de Torres y Santos [3], de Pérez y sus colaboradores [4], y de Gay y Berini [5]. Estos autores describen cómo realizar la técnica dependiendo de la pieza fracturada, así como también indican lo importante que es intentar primero la aplicación de la técnica cerrada, para disminuir el traumatismo; sin embargo, se debe acudir a la técnica antes mencionada, una vez agotadas las otras posibilidades.

La osteotomía fue necesaria utilizarla en el $16 \%$ de los casos en los que se fracturó una pieza dental en el transquirúrgico, en la clínica dental de la Universidad Iberoamericana, en el período febrero-mayo del 2013. Estos fueron casos en los que ya el levantamiento de un colgajo con osteotomía estaba seleccionado como alternativa de tratamiento, pero fue necesario ampliar la osteotomía para lograr la extracción del fragmento fracturado (figura 4).

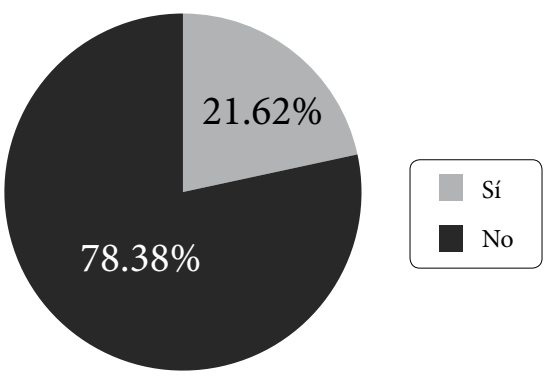

Figura 4. Ostectomía

Fuente: elaboración propia

Este resultado se sustenta con las investigaciones de Torres y Santos [3], de Hupp y sus colaboradores [6], y de Gay y Berini [5]. Sin embargo, el empleo de piezas de mano para la realización de osteotomía resulta ser peligroso, además de que conservar lo más íntegro po- sible el alvéolo, tras la extracción, es fundamental para motivos protésicos; por lo tanto, se debe sólo realizar osteotomía cuando sea estrictamente necesario y a cargo de profesionales que causen un mínimo desgaste a las paredes del alvéolo $[7,8]$.

El uso de un elevador apical fue necesario en el $24 \%$ de los casos en los que se fracturó una pieza dental en el transquirúrgico, en la clínica dental de la Universidad Iberoamericana (figura 5).

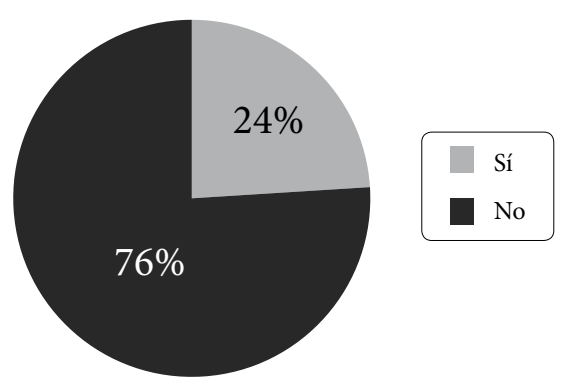

Figura 5. Uso de elevador apical

Fuente: elaboración propia

Estos resultados concuerdan con los estudios e investigaciones realizadas por Pérez y sus colaboradores [4], por Hupp y sus colaboradores [6] y por Águila [9]. Sin embargo, Gay y Berini [5] indican que el empleo de estos instrumentos debe suceder sólo si existe una gran posibilidad de que esta técnica dé resultados positivos para la extracción del fragmento, ya que puede causar daños al alvéolo, como por ejemplo, una alveolitis.

Por su parte, la odontosección fue la técnica de extracción más utilizada en el $38 \%$ de los casos en los que se fracturó una pieza dental en el transquirúrgico, en la clínica dental de la Universidad Iberoamericana, en el período febrero a mayo del 2013 (figura 6).

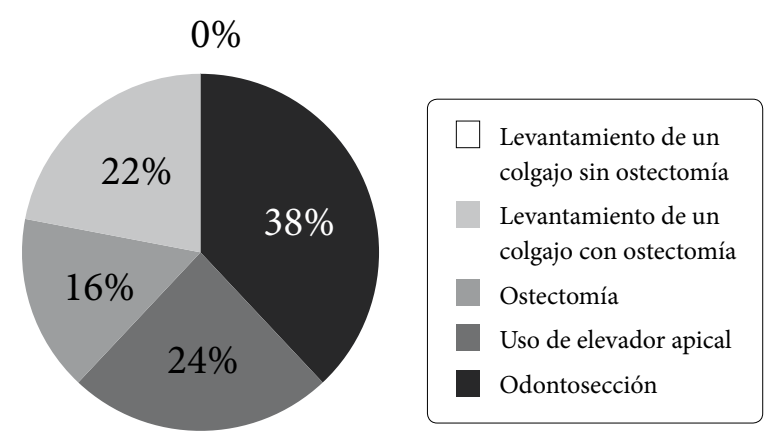

Figura 6. Técnicas más utilizadas Fuente: elaboración propia 
Esto concuerda con los estudios o investigaciones de Raspall [10], de Pérez y sus colaboradores [4], de Gay y Berini [5] y de Granados [11], quienes coinciden en que siempre que se fracture una pieza durante su extracción, la opción que se debe considerar en primera instancia debe ser la odontosección, la cual, si su empleo es posible, logrará dividir las raíces por método cerrado, por lo tanto facilitará la exodoncia de los fragmentos, disminuirá el traumatismo y garantiza un mejor posquirúrgico. Torres y Santos [3] y Hupp y sus colaboradores [6] consideran que la odontosección debe realizarse con piezas de mano de alta velocidad, con fresas adecuadas y con irrigación constante para disminuir las posibilidades de inflamación, dolor $\mathrm{y}$ trismo.

Fue necesario que el doctor responsable interviniera en el $73 \%$ de los casos en los que se fracturó una pieza dental en el transquirúrgico (figura 7).

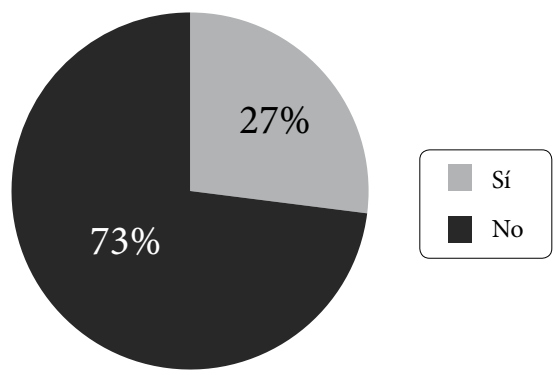

Figura 7. Necesidad de intervención del doctor responsable Fuente: elaboración propia

Estos resultados concuerdan con las investigaciones de Torres y Santos [3], de Navarro [8], de Hupp y sus colaboradores [6] y de López [1], quienes indican que ciertos procesos, como levantar un colgajo, extender osteotomías y realizar odontosección en piezas superiores, son técnicas delicadas, no precisamente indicadas para manos inexpertas, como solución a una complicación de este tipo; en caso de permitirlo, se debe mantener una constante vigilancia por parte del profesional. En las opiniones brindadas en la encuesta realizada a los doctores del área de cirugía de la Universidad Iberoamericana, expresan que la complejidad de la fractura y el nivel de clínica del estudiante serán los que determinarán si el estudiante es capaz o no de poseer la habilidad para resolver este tipo de complicación.

A la combinación de técnicas complementarias se recurrió en el 38\% de los casos en los que se fracturó una pieza dental en el transquirúrgico (figura 8).

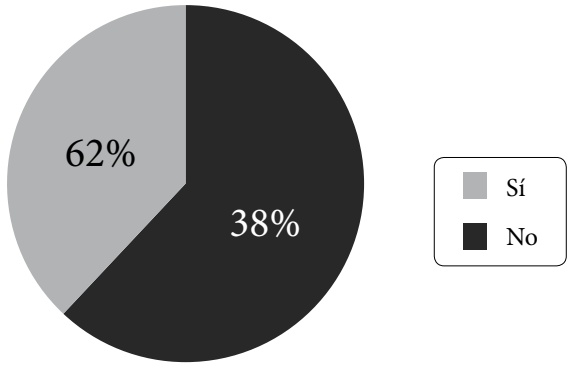

Figura 8. Combinación de técnicas

Fuente: elaboración propia

Gay y Berini [5] explican que, en ocasiones, dependiendo de las características de la fractura, una determinada técnica utilizada por sí sola puede no ser tan eficaz como la combinación adecuada con técnicas complementarias. En las opiniones brindadas en la encuesta realizada a los doctores del área de cirugía de la Universidad Iberoamericana, estos consideran que la selección de la técnica depende de las habilidades del operador, del nivel de la fractura y de la proximidad de esta a las estructuras anatómicas vecinas.

\section{Conclusiones}

Mediante la metodología empleada en esta investigación se pudo comprobar que la técnica de extracción de piezas fracturadas en el transquirúrgico que más se utilizó fue la odontosección, y la menos utilizada fue el levantamiento de un colgajo sin osteotomía; se determinó también que la intervención del doctor responsable para la extracción de las piezas fracturadas se presentó en mayor porcentaje en los casos en los en que no fue necesaria la intervención; así mismo, las técnicas de extracción de piezas fracturadas utilizadas en el área de cirugía de la clínica dental de la Universidad Iberoamericana fueron: la odontosección, en primer lugar, luego el uso de un elevador apical, seguido del levantamiento de un colgajo con osteotomía y de la extensión de la osteotomía en los casos en los que el levantamiento de un colgajo haya sido la opción predeterminada para el caso.

\section{Referencias}

[1] López Z. Complicaciones más frecuentes en la exodoncia. [Consultado: 21 de febrero de 2013]. Disponible en: http://www.uvsfajardo.sld.cu 
[2] Irureta MA. Cirugía dentomaxilar. [Consultado: 17 de marzo de 2013]. Disponible en: http://www.sdpt.net

[3] Torres L, Santos P. Respuesta clínica a la extracción quirúrgica de terceros molares clase II con pieza de alta y baja velocidad. Med Oral. 2007; 9(3): 71-4.

[4] Pérez JLG, Lagares DT, Calderón MG, Romero DG, Fernández LC, González EA. [Consultado: 17 de marzo de 2013]. Disponible en: http://www.ocwus.us.es

[5] Gay C, Berini L. Tratado de cirugía bucal. España: Ergón; 2011.

[6] Hupp J, Ellis III E, Tucker M. Cirugía oral y maxilofacial contemporánea. 5ª ed. Barcelona: Elsevier Mosby; 2010.

[7] Gómez M, Peñarrocha M, García B. Extracción de resto radicular con técnicas de ventana vestibular y colocación simultánea de dos implantes. Rev Period. 2005; 15: 165-70.

[8] Navarro C. Cirugía oral. España: Aran; 2008.

[9] Águila Y. Complicaciones inmediatas de la extracción dentaria. [Consultado: 17 de marzo de 2013]. Disponible en: http://www.portalesmedicos.com/publicaciones/articles/3273/4/Complicaciones-inmediatas-de-la-extraccion-dentaria

[10] Raspall G. Cirugía oral e implantología. 2. ed. Madrid: Editorial Médica Panamericana; 2007.

[11] Granados D. Cirugía oral. Exodoncia. [Consultado: 7 de abril de 2013]. Disponible en: http://www.icctsaludoral.com 